\title{
Après MARCKS, p53 augmente le Capital de la protéine kinase $C$
}

C'cst un ballet d'un nouveau type dont médecine/sciences veut présenter l'argument à ses lecteurs. Certains des personnages sont probablement nouveaux pour les fidèles de $\mathrm{m} / \mathrm{s}$ et doivent, de ce fait, être présentés. MARCKS (myristoylated alanine-rich $C$ kinase substrate) est l'un des substrats majeurs de la protéine kinase C. Il s'agit d'une protéine inhabituellement riche en alanine, glycine, proline et acide glutamique, ayant une structure en bâtonnet, composée de trois domaines : une région aminoterminale myristoyléc, responsable de la fixation à la membrane ; un domaine central conscrvé et une région basique contenant des sites de phosphorylation par la protéine kinase $\mathrm{C}$ et de liaison de la calmoduline et de l'actine. Cette extrémité effectrice carboxytcrminalc a une structure d'hélice $\alpha$-amphipatique.

MARCKS, ainsi qu'unc autre moléculc de la même famille mais de localisation différente (Mac MARCKS/ F52), semblent jouer un rôle important dans la régulation de la plasticité du cytosquelette et, notamment, des filaments d'actinc. Le modèle privilégić à l'heure actuelle est celui d'une protéine liée à la membrane de cellules au repos. Dans ces conditions, et à basse concentration en calcium cytoplasmique, une molécule MARCKS interagit avec deux filaments d'actinc, les liant l'un à l'autre et assurant ainsi une plus grande rigidité des faisceaux d'actine liés à la membrane. Une augmentation de la concentration en calcium intracellulaire, provoquée par différents effecteurs, conduit à la liaison du complexe calmoduline/ $\mathrm{Ca}^{2+} \mathrm{au}$ site effecteur carboxyterminal; sous cette forme, la molécule de MARCKS n'interagit plus qu'avec un scul filament d'actine, ce qui aboutit à unc moins grande rigidité du cytosquelette cellulaire. Dans d'autres situations où un effectcur extracellulaire met en jeu la voie passant par la protéine kinase $\mathrm{m} / \mathrm{s} n^{\circ} 2$ vol. 9, féurier 93
C, et à basse concentration en calcium intracellulaire, la phosphorylation du domaine carboxyterminal de MARCKS s'accompagne de son détachement de la membrane et de sa liaison à un seul filament d'actine. Ces conditions sont propices à un remaniement très prof ond du cytosquelette tel qu'il apparaît dans diverses situations, par exemple la mobilité cellulairc ou la transformation maligne. Les activateurs de la protéine kinase $\mathrm{C}$ étant des promoteurs de la transformation tumorale, cela peut donc constituer l'une des voies par lesquelles les signaux prolifératifs aboutissent à une modification du cytosquelette ([1] et figure 1).

Il existe une exclusion entre la liaison de la protéinc MARCKS à des protéines fixant le calcium, telle la calmoduline, et son aptitude à ctre phosphorylée par la protéine kinasc C. Une autre calciprotéine, appelée S100b, a un pouvoir inhibitcur cncore plus important que la calmoduline sur la phosphorylation de MARCKS.

D'autres personnages de notre ballet sont beaucoup plus familliers à nos lecteurs, par excmple p53, un régulatcur négatif de la prolifération cellulaire $(\mathrm{m} / \mathrm{s}$ $n^{\circ}$ 1, vol. 9, p. 79).

p53 est un facteur de transcription dont la translocation dans le noyau cst soumise à différents systèmes de régulation, parmi lesquels la phosphorylation par différentes protéines kinases, la kinase lice au cycle cellulairc p34 ${ }^{\text {cdc2 }}$, la casćine kinase II et la protéinc kinase C. La forme active de p53 semble ĉtre oligomérique. D'un point de vuc structural, p53 comporte une région carboxyterminale basique étendue pouvant adopter une conformation en hélice $\alpha$-amphipatique, domaine probablement impliqué dans la liaison à l'ADN et dans la formation des oligomères stables. T.R. Hupp et al. du laboratoire de D.T. Vane (Dundee, Écosse) viennent de montrer que la liaison de p53 à l'ADN pouvait ĉtre contrôléc par différentes modifications de cettc extrémité carboxyterminalc. I a protéine recombinante normale se lic en effet extrêmement mal à sa séquence cible d'AI)N (m/s $n^{\circ} 7$, vol. 8, p. 732). I a liaison peut être activéc par un anticorps monoclonal se liant au domaine carboxyterminal. Un résultat similaire peut être obtenu par différents moyens : utilisation de p53 ayant une délétion carboxyterminale, protéolyse ménagéc, action de la protéine dnaK de $E$. coli, ou phosphorylation de l'extrémité carboxyterminale par la caséine kinase 1I. Tous ces résultats identifient le domaine carboxyterminal de p53 comme un site potentiel de régulation de sa lonction, par l'intermédiaire de modifications covalentes post-traductionnelles (protéolyse, phosphorylation) ou par celui de modification de conformation induite par interaction avec d'autres facteurs cellulaires encore non caractérisés. I'’action de la dnaK de $E$. coli est cohérente avec ce modèle puisqu'il s'agit d'unc protéine chaperon susceptible de modifier des interactions inter-protéiques.

L'examen attentif par J. Baudier et al., du laboratoire de J.J. I awrence (INSERM, Centre d'Etudes Nucléaires, Grenoble, France) du domainc carboxyterminal de p53 lui a révélé des similitudes structurales et de séquences avec la région catalytique de MARCKS. I)c fait, ces autcurs ont démontré que, comme MARCKS, p53 était un substrat de la protéine kinasc $\mathrm{C}$, in vivo et in vitro, et fixait, en présence de calcium, la protéine $\mathrm{S} 100 \mathrm{~b}$ [3]. Unc interaction très faible a également été démontréc avec la calmoduline. La fixation de p53 à S100b inhibe sa phosphorylation par la protéine kinase $\mathrm{C}$ et son oligomérisation. Mieux même, S100b est capable d'entraîner la dissociation des oligomères de p53. Il existe ainsi un antagonisme entre le calcium et des protéines liant le calcium type calmoduline
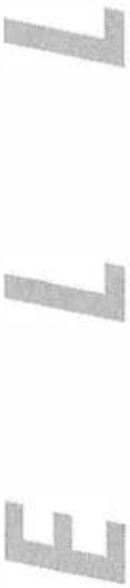


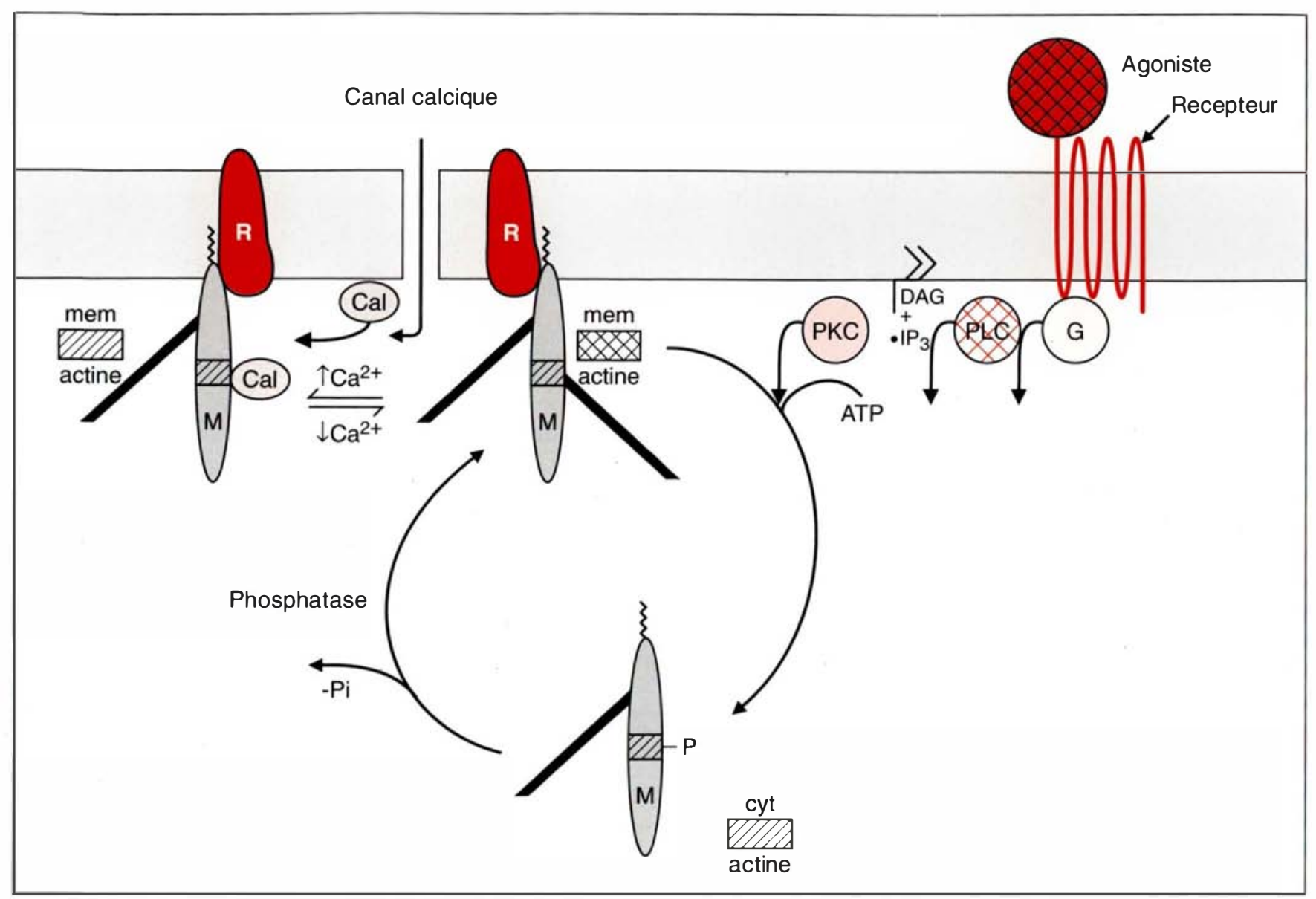

Figure 1. Modèle de la régulation de la rigidité du cytosquelette par MARCKS, la protéine kinase C et la calmoduline/ $\mathrm{Ca}^{2+}$. Au repos, MARCKS $(\mathrm{M}$, ovale gris) est lié à la face interne de la membrane plasmique par son résidu myristoyl (ligne brisée) et, à bas $\mathrm{Ca}^{2+}$ intracellulaire, assure la rigidité du cytosquelette en connectant deux fibres d'actine membranaire (mem-actine). Une augmentation du $\mathrm{Ca}^{2+}$ intracellulaire, suite, par exemple, à l'activation de récepteurs couplés à des canaux calciques (directement ou indirectement), entraine la fixation du complexe calmoduline/Ca2+ (Cal) sur MARCKS qui ne peut plus alors fixer qu'une fibre d'actine, ce qui aboutit à une diminution de la rigidité du cytosquelette et de son ancrage à la membrane plasmique. L'activation de récepteurs couplés par des protéines $G$ (G) à la phospholipase $C(P L C)$ et, par l'intermédiaire du diacylglycérol (DAG, produit de l'hydrolyse par la PLC du phosphatidyl inositol 4,5 P2 en DAG et en inositol 3 phosphate, IP3), conduit à la phosphorylation de MARCKS. Ainsi modifiée, cette protéine devient insensible au calcium et aux calciprotéines et se détache de la membrane, ne pouvant rester fixée qu'à une fibre $d^{\prime}$ actine cytoplasmique (cyt actine). Cette dernière condition permet un réarrangement profond de l'architecture de cytosquelette. (D'après [1].

et $\mathrm{S} 100 \mathrm{~b}$, et la protéine kinase $\mathrm{C}$ pour la régulation de l'état structural dans lequel se trouve p53. Cet antagonisme coïncide avec les différentes situations mettant en jeu ces deux types de modulateurs. S100b semble être synthétisée en phase G1, dans la cellule au repos, alors que la protéine kinase $\mathrm{C}$ est activée par des agents mitogéniques. Une ambiguïté persiste néanmoins, à la lumière de ces informations, sur l'influence de l'état d'oligomérisation sur la fonction de p53.
La forme active de cette protéine est oligomérique, mais des formes multimériques de haut poids moléculaire semblent être inactives et on connaît mal les mécanismes de la transition multimères inactifs $\rightarrow$ oligomères actifs. Celle-ci pourrait être catalysée par les mécanismes décrits par Hupp et al. (phosphorylation par la caséine kinase II, changement de conformation de l'extrémité carboxyterminale) [2], alors que l'action de la protéine kinase $\mathrm{C}$, dont le résidu cible sur p53 n'est pas encore connu avec précision, pourrait être de stabiliser les oligomères actifs, de les protéger contre la dissociation inactivante provoquée par les calciprotéines et le calcium [3]. Il faut remarquer que, quoique étant un régulateur négatif de la prolifération cellulaire, p53 semble être normalement soumis à des influences inactivatrices dans les cellules au repos, en G1 (protéine kinase $\mathrm{C}$ inactive, S100b élevée), alors que son pouvoir antiprolifératif, lié à sa fixation à l'ADN $\left(\mathrm{m} / \mathrm{s} n^{\circ} 7\right.$, vol. 8 , 
p. 732) est stimulé dans des conditions d'induction mitotique (activation de la caséine kinase II et de la protéine kinase C). Cela confirme les données de l'inactivation homozygote du gène p53 par recombinaison homologue, qui n'empêche pas un développement tout à fait normal des souris, indiquant que p53 n'est pas indispensable à la régulation du cycle cellulaire $\left(m / s \quad n^{\circ} 5\right.$, vol. 8, p. 492). En fait, dans les cellules normales, p53 pourrait surtout jouer un rôle de limitateur d'une prolifération excessive : inactif en l'absence de mitose, il serait activé lors de l'induction de la prolifération pour la limiter. Un tel schéma expliquerait que l'absence de ce contrôleur des proliférations "pathologiques" conduise à l'émergence de cancers, d'autant plus qu'un autre rôle de p53 semble être de bloquer les cellules en phase G1 lorsqu'existent des lésions de l'ADN ou en présence d'agents mutagènes $(\mathrm{m} / \mathrm{s}$ $n^{\circ}$ 9, vol. 8, p. 1002 et $n^{\circ}$ 1, vol. 9, p. 9), évitant ainsi la "fixation " dans le génome d'altérations génétiques potentiellement transformantes.

\section{A.K.}

1. Aderem A. The MARCKS brothers: a family of protein kinase C substrates. Cell 1992 ; $71: 713-6$.

2. Hupp 'TR, Meek DW, Midgley CA, Lane DP. Regulation of the specific DNA binding function of p53. Cell 1992; 71: 875-86.

3. Baudier J, Delphin C, Grunwald D, Khech-

bin S, Lawrence J.J. Characterization of the

turnor suppressor protein $\mathrm{p} 53$ as a protein kinase
$\mathrm{C}$ substrate and a S100b binding protein. Proc Natl Acad Sci USA 1992 ; 89 : 11627-31. 